\title{
Sample Return from the Moon's South Pole-Aitken Basin
}

\author{
A White Paper submitted to the 2023 \\ Planetary Science Decadal Survey, July 15, 2020
}

Co-authors

Brad Jolliff, Washington University in St. Louis, bjolliff@wustl.edu

Noah Petro, NASA GSFC, noah.e.petro@nasa.gov

Daniel Moriarty, NASA GSFC/USRA, daniel.p.moriarty@nasa.gov

Ryan Watkins, PSI, rclegg-watkins@psi.edu

James Head III, Brown University, james_head@brown.edu

Ross Potter, Brown University, ross_potter@,brown.edu

Co-signatories on the last page

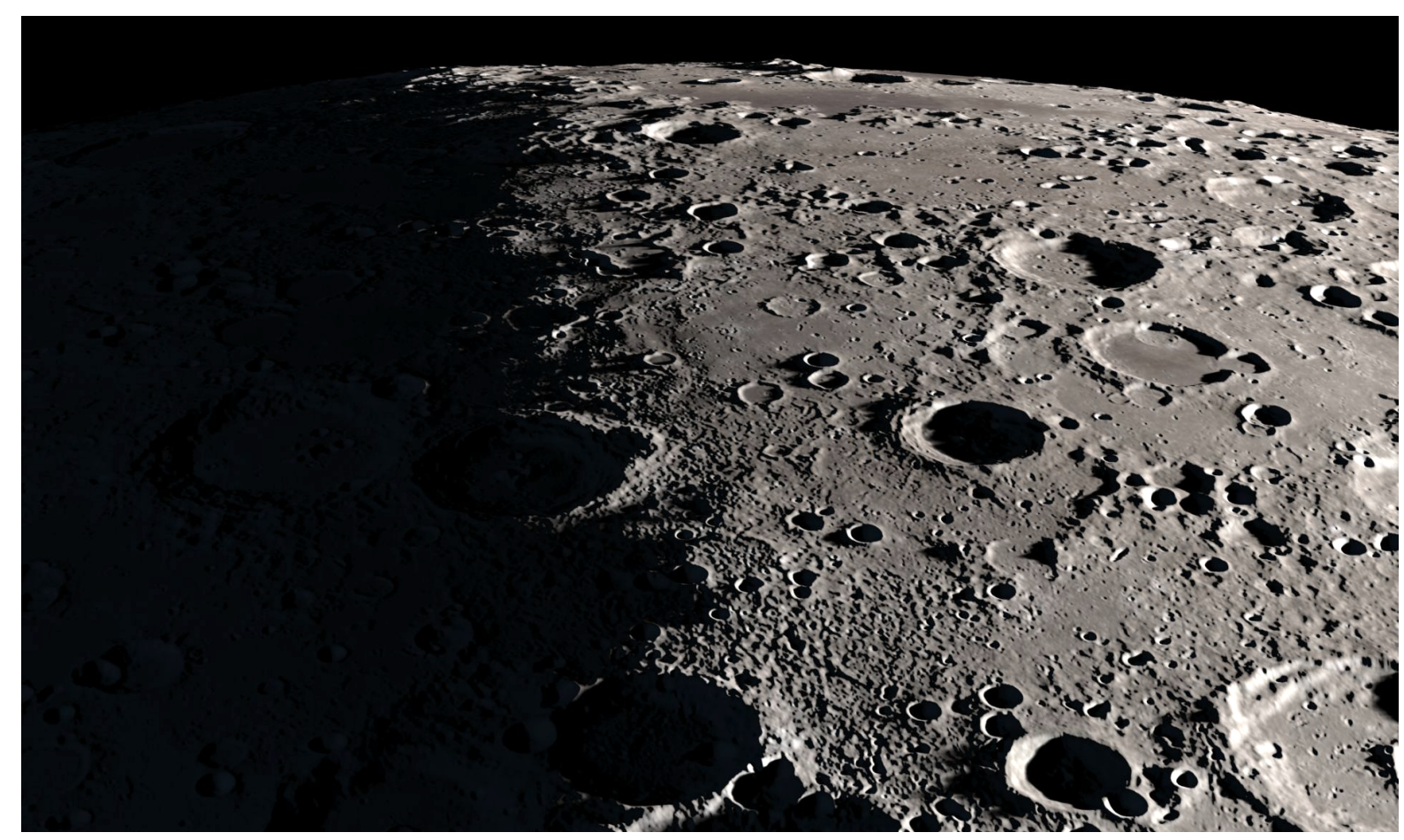

Oblique view of central South Pole-Aitken basin along day-night terminator derived from Lunar Reconnaissance Orbiter data [NASA/GSFC/ASU] (credit: Ernie Wright, GSFC SVS)]. SPA materials reveal the rich geologic history of the basin, with profound implications for the early Earth, Moon, and the Solar System. SPA samples are needed to fully address these diverse and fundamental science questions! 
The Moon's enormous South Pole-Aitken basin (SPA) holds in its rocks and regolith keys to unlock secrets of the early evolution of the Moon, Earth, and Solar System. Was there a cataclysmic bombardment of the inner Solar System $\sim 4$ billion years ago, and if so, what caused it? Did the impact that formed the SPA basin cause widespread magmatic activity that is recorded in its ancient rocks? Did the impact excavate materials from the Moon's mantle and what can those materials, as well as later-formed volcanic rocks, reveal about the nature of the Moon's early differentiation and formation of its planetary-scale asymmetries? To answer these questions, it is critical to collect and return samples from the interior of SPA and analyze the samples in the best laboratories on Earth. Accordingly, sample return from SPA remains a high science priority and a key test of hypotheses regarding some of the most fundamental aspects of the early history of the Solar System, with implications for our understanding of environments on the early Earth. Past mission proposals indicate that a New Frontiers scale is appropriate for SPA sample return. While the possibility of a human mission to the South Pole of the Moon is compelling as it may provide the opportunity to sample materials ejected from the SPA basin, such missions may not directly sample the key material needed to determine unambiguously the basin's age.

\section{Why Do We Need Samples From The Moon's South Pole-Aitken Basin?}

The Moon preserves an unparalleled and readily accessible record of early Solar System impact bombardment, and the SPA basin on the Moon's southern farside is a key scientific target because it is the largest and oldest well-preserved impact structure on the Moon. As such, its age and the ages of subsequent large impacts within SPA will provide a new understanding of early Solar System events relating to heavy impact bombardment during the first $\sim 500$ million years following accretion of the planets. Chemical, mineralogical, and chronological analysis of SPA samples can resolve fundamental questions about the evolution of the early Solar System, including firm constraints on models for migration of giant-planet orbits during the early epoch of Solar System history (Gomes et al., 2005; Marchi et al., 2012). SPA samples will answer significant questions about the early evolution of the Moon and, by analogy, the terrestrial planets. The science associated with this mission concept is among the highest priority for Solar System exploration as laid out by two previous Planetary Science Decadal Surveys (NRC, 2003; NRC, 2011).

Samples from SPA will provide a test of the "cataclysm" or late-heavy bombardment that is implied by the analysis of lunar samples (Tera et al., 1974). Characteristics of planetary systems determined from the Kepler mission as well as other astronomical planet-finding data, integrated with recent advances in concepts for early Solar System history, are reshaping how we think about early Solar System evolution. It is possible that migration of the giant planets affected the configuration of the inner Solar System as well as the positioning of the ice giants (e.g., Nice and Grand Tack models, summarized by Bottke and Norman, 2017). Determining the chronology of SPA basin on the Moon is one of the few firm observational constraints that can be brought to bear on these models.

The SPA impact event was enormous, forming a basin some $2200 \times 2500 \mathrm{~km}$ in size (GarrickBethel \& Zuber, 2009) and excavating materials from great depth, potentially including both crust and mantle materials (Potter et al., 2012). Its effects on the Moon were global, forming a Moonwide stratigraphic event; determining the age of formation will bracket the ages of other ancient, Pre-Nectarian basins on the Moon as well the origin of the apparent episode of igneous activity around 4.35 Ga (Schultz and Crawford, 2015; Kring et al., 2015; Borg et al., 2015; Shearer et al., 
2015). Materials from SPA basin hold a record of the process of very large impact-basin formation and will serve as ground truth for remote sensing of SPA basin materials (Melosh et al., 2017). Despite decades of study, the composition and nature of the lunar mantle is still uncertain, and SPA-SR could uniquely address this question. Volcanic rocks from the SPA interior will contribute to our understanding of basin evolution and chronology, as well as mantle heterogeneity. Paleomagnetic analyses will provide the oldest constraints on the lunar core dynamo, aiding our understanding of the Moon's earliest thermal evolution.

GRAIL data provided an important revision to ideas about the Moon's crust, relevant to SPA basin. It is very likely that the SPA impact formed a transient cavity extending into the Moon's upper mantle, and the impact melt that filled the basin is a mixture of mantle and crustal materials. Recent petrologic and geochemical modeling studies suggest that this impact-melt body differentiated, with mineralogical separations that explain the present-day distribution of rocks at the surface near the center of the basin. These materials were later excavated by impacts into the SPA basin. This idea represents an important new hypothesis and a potential paradigm shift that is testable by sample return from the interior of the SPA basin.

Remote sensing missions of the past decade, especially he Moon Mineralogy Mapper $\left(\mathrm{M}^{3}\right)$ on Chandrayaan-1, and the ongoing LRO mission, permit a new level of scientific and safety assessment of potential landing sites within SPA Basin. Timing and scientific context for the SPA basin sample return continue to be excellent and, in fact, even better than at the time of the last Planetary Science Decadal Survey.

Chronology: Unravelling the impact chronology recorded by complex impact-melt rocks and breccias requires analysis of samples in the best terrestrial laboratory conditions and by modern, highly precise analytical methods. Coordinated petrographic and chronologic studies are required to understand age data and to distinguish, for example, crystallization vs. impact ages. Multiple chronometers provide data on systems with different closure and diffusion properties. The impact that produced the SPA basin melted an enormous volume of rock and reset the age of a large part of the Moon. Analyses of many of the thousands of small rocklets associated with sample return would define the age of the SPA melt and potentially of a distribution of younger ages from the later, large impacts within SPA. Such age determinations will clarify whether the mechanisms proposed to account for the observed lunar cataclysm from Apollo samples are compatible with the farside SPA samples. These samples will provide a record of the duration and timing of basin impacts on the Moon as well as a key absolute calibration point for the pre-4.0 Ga chronology (Hiesinger et al., 2012; Van der Bogert et al., 2017).

The impact cataclysm hypothesis was first indicated by clustering of ages and the identification of large-scale mobilization of $\mathrm{Pb}$ at $\sim 3.9 \mathrm{Ga}$ (Tera et al., 1974). The $3.9 \mathrm{Ga}$ age was also identified in Ar-Ar chronology (Turner et al., 1977). The hypothesis was further supported by $2.9 \mathrm{Ga} \mathrm{Rb-Sr}$ ages of impact melt rocks (Papanastassiou and Wasserburg, 1971). The Sm-Nd system is also an essential analytical method for lunar chronology because it is least disturbed by later impact events (Norman et al., 2016). This system is important because SPA is old and has undergone later impacts that likely have disturbed other systems. Because of this differential response of the chronometers to impact heating and alteration, samples from the SPA basin must be analyzed by all these techniques in laboratories on the Earth. High precision methods (TIMS: Rb-Sr, Sm-Nd, UPb; ICPMS: Lu-Hf; and Ar-Ar) will be needed for bulk and carefully separated materials, and in- 
situ analyses such as secondary ion mass spectrometry (SIMS) will be conducted on mineral grains including zircon (e.g., Grange et al., 2013), other Zr- and U-rich minerals, and phosphates (Merle et al., 2014). These approaches, when used together, provide the critical data to unravel the impact history and evolution of SPA.

Impact Basin Formation Processes: Sophisticated numerical modeling of the SPA impact event has been the object of several recent studies (e.g., Potter et al., 2012; Melosh et al., 2017). These models will be further constrained with additional sample data. The age of SPA formation will constrain the thermal state of the Moon's lithosphere at the time of the impact. Samples will reveal the rock types and compositions of impact melt produced by the event and subsequent potential differentiation of the melt volume. Clasts in breccia and geochemical mixing analysis using clast compositions will reveal target lithologies, including deep crustal and mantle components. The compositional "anomaly" that still exists in the interior of SPA reflects a mafic (Fe-rich) composition (Lawrence et al., 2007). However, until we have direct samples of the materials that give rise to the compositions, we will not know fully how to interpret them. The same holds for the mineralogical signatures seen in orbital spectroscopic data (e.g., Lucey et al., 1998; Pieters et al., 2001). Recent mapping of mafic mineral signatures in crater central peaks and elsewhere (Moriarty et al., 2013; Moriarty and Pieters, 2018) reflects a mixture of SPA substrate lithologies produced by differentiation of the melt sheet (Vaughan and Head, 2013; Hurwitz and Kring, 2014; Cassanelli and Head, 2016) and later volcanic rocks. Samples are essential to provide ground truth for the orbital data.

Thermal Evolution of the Moon. Samples will be used to investigate sources and distribution of Th and other heat-producing elements to understand the Moon's differentiation and thermal evolution. Orbital measurements show a modest Th anomaly associated with the SPA interior, and it is possible that SPA is old enough to have occurred prior to the final migration and solidification of KREEP-rich materials. Coupling this signature with identification of host lithologies and age will enable new tests of models of lunar differentiation and the origin of the Moon's prominent asymmetries, and of whether the orbital SPA Th signature is associated with differentiation of the SPA impact melt body (Hurwitz and Kring, 2014) or excavation of late-stage LMO dregs (Moriarty et al., 2019).

Volcanic Components. SPA did not apparently undergo basaltic filling and resurfacing to the same extent as nearside basins such as Imbrium. Nonetheless, volcanic rocks occur in the interior of the basin as exposed mare basalts, buried "cryptomare" (Yingst and Head, 1999; Whitten and Head, 2015), and non-mare emplacements (Moriarty and Pieters, 2015; 2018). Analysis of Apollo regolith samples indicates that lateral transport would have introduced fragments of these rock types to regolith in varying degrees everywhere in SPA. Ages and compositions of SPA magmas will allow us to determine how mantle source regions (composition, volatiles, mineralogy, thermal history, depth of melting, model ages) on the Moon's far side compare to basalts sampled by Apollo, Luna, and lunar meteorites. A sample location near the center of SPA should include rock materials from "Mons Marguerite" (formerly Mafic Mound), which may be an ancient volcanic construct associated with the formation of SPA and the evolution of its melt sheet (Moriarty and Pieters, 2015). Isotopic, geochemical, and mineralogical analysis of these volcanic materials will help determine global mantle heterogeneity, the nature, timing, and extent of primordial lunar differentiation, and post-differentiation magmatic/thermal evolution of the mantle (e.g., overturn of magma ocean cumulates). 
Volatile-element contents. New discoveries in lunar samples and remote sensing have emphasized the importance of volatiles - both exogenous and endogenous - in the Moon's history. Materials excavated from deep in the Moon will offer the opportunity to investigate endogenous volatiles in a variety of new and different kinds of lithologies relative to Apollo and Luna samples. Agglutinates in central SPA should contain a record of more southerly surface volatiles, and potentially exogenous volatile-bearing deposits. Stable isotope compositions of samples record processes as well as origin. Modern analytical methods (e.g., SIMS on individual grains) minimize required sample mass and improve detection limits (Shearer and Borg, 2006; Hashizume \& Chaussidon, 2009). Coupled with chronology, results provide time-stamped volatile information in both magmatic and surface samples.

Implementation: Solar System Chronology remains among the highest priority lunar-focused missions owing to the unparalleled record of early cataclysmic bombardment of the Earth-Moon system and the inner Solar System (Cohen et al., 2020). Multiple implementations certainly exist to address the issue. In this white paper, we present the case for sample return as the most efficient way to unambiguously determine the chronology of the SPA basin. As a concept that has actually been proposed three times $(2005,2010,2016)$ to the New Frontiers program and that was runner up to the selected mission twice (Phase A Concept Study Reports, 2006; 2011), we describe briefly the implementation associated with that mission. The MoonRise mission concept proposed to land in the center of the SPA basin and sample rock materials in the regolith by scooping and sieving to extract a kilogram or more of rock material, amounting to many thousands of small rock fragments. Over $100 \mathrm{~g}$ of bulk regolith was also planned to be collected. This approach would leverage the natural impact process, which has, over the millennia, delivered a variety of local rock materials to any given site while preserving the original local material in the regolith. The efficiency of this process is well documented in the Apollo samples, and impact crater observations and theory support the effectiveness of ballistic sedimentation in producing well mixed and diverse deposits.

Indeed, the process of excavation and ejection of materials from impact craters renders the South Pole of the Moon, which lies on the southern rim of SPA, a place where samples could be collected that would address many of the SPA sample return objectives, especially if collected by astronauts. Artemis will in fact be a great complementary mission or missions to SPA sample return, and samples from the two locations, center of the basin and a rim location near the South Pole, would retire many of the risks associated with having a sample of SPA only from the interior.

Once samples from the SPA interior are in hand, we will connect their characteristics with remote sensing data sets to better understand a larger portion of the basin. Indeed we will use what is learned from SPA samples to identify lunar meteorites or Apollo fragments likely from SPA (e.g., Garrick-Bethell et al. 2020). Assuming Artemis missions go to the South Pole, those samples will provide an excellent point of comparison to those collected from the interior of the basin, and would allow, for the first time, a coordinated analysis of the differences of how material from a basin are excavated, melted, and emplaced. The integration of these data will lead synergistically to a significantly improved understanding of the SPA basin, its materials, and what they have to reveal about the early Moon and entire Solar System. 


\section{References}

Borg, L. E., A. M. Gaffney, C. K. Shearer (2015) A review of lunar chronology revealing a preponderance of 4.34-4.37 Ga ages. Meteorit. Planet. Sci. 50, 715-732.

Bottke, W. F., M. D. Norman (2017) The late heavy bombardment. An. Rev. Earth Planet. Sci. 45, 619-647.

Cassanelli, J. P., J. W. Head (2016) Did the Orientale impact melt sheet undergo large-scale igneous differentiation by crystal settling? Geophys. Res. Lett. 43, 11156-11165.

Cohen, B., S. Lawrence, B. Denevi, T. Glotch, D. Hurley, C. Neal, M. Robinson, R. Watkins, and R. Weber (2020) Lunar missions for the decade 2023-2033, A White Paper submitted to the 2023 Planetary Science Decadal Survey.

Garrick-Bethel, I., M. T. Zuber (2009) Elliptical structure of the lunar South Pole-Aitken basin. Icarus 204, 399-408.

Garrick-Bethell, I., K. Miljković, H. Hiesinger, C. H. van der Bogert, M. Laneuville, D. L. Shuster, and D. G. Korycansky (2020), Troctolite 76535: A sample of the Moon's South Pole-Aitken basin?, Icarus, 338, 113430.

Gomes, R., K. Tsiganis, A. Morbidelli, H. F. Levison (2005) Origin of the cataclysmic Late Heavy Bombardment period of the terrestrial planets. Nature 435, 466-469.

Grange, M. L., R. T. Pidgeon, A. A. Nemchin, N. E. Timms, C. Meyer (2013) Interpreting $\mathrm{U}-\mathrm{Pb}$ data from primary and secondary features in lunar zircon. Geochim. Cosmochim. Acta 101, 112-132.

Hashizume, K., M. Chaussidon (2009) Two oxygen isotopic components with extra-selenial origins observed among lunar metallic grains - In search for the solar wind component. Geochim. Cosmochim. Acta 73, 30383054.

Hiesinger, H., C. H. van der Bogert, J. H. Pasckert, N. Schmedemann, M. S. Robinson, B. Jolliff, N. Petro (2012) New crater sizefrequency distribution measurements of the South Pole-Aitken basin. Lunar Planet. Sci 43, \#2863.
Hurwitz, D. M., D. A. Kring (2014) Differentiation of the South Pole-Aitken basin impact melt sheet: Implications for lunar exploration. J. Geophys. Res. 119, 1110-1133.

Kring, D. A., P. J. McGovern, R. W. K. Potter, G. S. Collins, M. L. Grange, A. A. Nemchin (2015) Was an epoch of lunar magmatism triggered by the South Pole-Aitken basin impact? Workshop on Early Solar System Impact Bombardment III. Lunar \& Planetary Inst., Houston, TX, p. \#3009.

Lawrence, D. J., R. C. Puetter, R. C. Elphic, W. C. Feldman, J. J. Hagerty, T. H. Prettyman, P. D. Spudis (2007) Global spatial deconvolution of Lunar Prospector Th abundances. Geophys. Res. Lett. 34, L03201.

Lucey, P. G., G. J. Taylor, B. R. Hawke, P. D. Spudis (1998) $\mathrm{FeO}$ and $\mathrm{TiO}_{2}$ concentrations in the South Pole-Aitken basin: Implications for mantle composition and basin formation. J. Geophys. Res. 103, 3701-3708.

Marchi, S., W. F. Bottke, D. A. Kring, A. Morbidelli (2012) The onset of the lunar cataclysm as recorded in its ancient crater populations. Earth Planet. Sci. Lett. 325-326, 2738.

Melosh, H. J., J. Kendall, B. Horgan, B. C. Johnson, T. Bowling, P. G. Lucey, G. J. Taylor (2017) South Pole-Aitken basin ejecta reveal the Moon's upper mantle. Geology 45, 1063-1066.

Merle, R. E., A. A. Nemchin, M. L. Grange, M. J. Whitehouse, R. T. Pidgeon (2014) High resolution $\mathrm{U}-\mathrm{Pb}$ ages of $\mathrm{Ca}$-phosphates in Apollo 14 breccias: Implications for the age of the Imbrium impact. Meteorit. Planet. Sci. 49, 2241-2251.

Moriarty, D. P. I., C. M. Pieters (2015) The nature and origin of Mafic Mound in the South Pole-Aitken Basin. Geophys. Res. Lett. 42, 7907-7915.

Moriarty III, D. P., C. M. Pieters (2018) The character of South Pole-Aitken Basin: Patterns of surface and subsurface composition. J. Geophys. Res. 123, 729-747. 
Moriarty, D. P., C. M. Pieters, P. J. Isaacson (2013) Compositional heterogeneity of central peaks within the South Pole-Aitken Basin. J. Geophys. Res. 118, 2310-2322.

Moriarty, D. P., R. N. Watkins, S. N. Valencia, J. D. Kendall, \& N. E. Petro (2019) Mineralogy of thorium-enhanced materials within the South Pole-Aitken Basin: Possible traces of the lunar upper mantle. In Lunar Planet. Sci. 50, \#2874.

Norman, M. D., L. A. Taylor, C.-Y. Shih, L. E. Nyquist (2016) Crystal accumulation in a 4.2 Ga lunar impact melt. Geochim. Cosmochim. Acta 172, 410-429.

NRC (2003) New Frontiers in the Solar System: An Integrated Exploration Strategy. National Academies Press, Washington, DC.

NRC (2011) Vision and Voyages for Planetary Science in the Decade 2013-2022. National Academies Press, Washington, DC.

Papanastassiou, D. P., G. J. Wasserburg (1971) Lunar chronology and evolution from $\mathrm{Rb}-\mathrm{Sr}$ studies of Apollo 11 and 12 samples. Earth Planet Sci. Lett. 11, 37-62.

Pieters, C. M., J. W. Head, III, L. Gaddis, B. L. Jolliff, M. Duke (2001) Rock types of the South Pole-Aitken basin and extent of basaltic volcanism. J. Geophys. Res. 106, 2800128022.

Potter, R. W. K., G. S. Collins, W. S. Kiefer, P. J. McGovern, D. A. Kring (2012) Constraining the size of the South Pole-Aitken basin impact. Icarus 220, 730-743.

Schultz, P. H., D. A. Crawford (2015) SPAimpact origin for the nearside dike system on the Moon. Lunar Planet. Sci. 46, \#2416.
Shearer, C. K., S. M. Elardo, N. E. Petro, L. E. Borg, F. M. McCubbin (2015) Origin of the lunar highlands Mg-suite: An integrated petrology, geochemistry, chronology, and remote sensing perspective. Amer. Mineral. 100, 294-325.

Shearer, C. K., L. E. Borg (2006) Big returns on small samples: Lessons learned from the analysis of small lunar samples and implications for the future scientific exploration of the Moon. Chemie der Erde 66, 163-185.

Turner, G. (1977) Potassium-argon chronology of the Moon. Phys. Chem. Earth 10, 145195.

Van der Bogert, C., H. Hiesinger, R. Z. Povilaitis, M. S. Robinson, H. Meyer, L. R. Ostrach (2017) Regional lunar stratigraphy derived from CSFDs extracted from the $>5 \mathrm{~km}$ global crater catalog. Lunar Planet. Sci. 48, \#1437.

Vaughan, W. M., J. W. Head (2014) Impact melt differentiation in the South Pole-Aitken basin: Some observations and speculations. Planetary and Space Science 91, 101-106.

Whitten, J. L., J. W. Head (2015) Lunar cryptomaria: Physical characteristics, distribution, and implications for ancient volcanism. Icarus 247, 150-171.

Yingst, R. A., J. W. Head, III (1997) Volumes of lunar lava ponds in South Pole-Aitken and Orientale basins: Implications for eruption conditions, transport mechanisms, and magma source regions. J. Geophys. Res. 102, 10909-10931.

\section{Co-Authors}

Brad Jolliff, Washington University in St. Louis, bjolliff@,wustl.edu

Noah Petro, NASA GSFC, Noah.E.Petro@nasa.gov

Daniel Moriarty, NASA GSFC/USRA, daniel.p.moriarty@nasa.gov

Ryan Watkins, PSI, rclegg-watkins@psi.edu

James Head III, Brown University, james head@brown.edu

Ross Potter, Brown University, ross_potter@brown.edu

\section{Co-Signatories}

Barbara Cohen, GSFC, Barbara.A.Cohen@nasa.gov

William Bottke, SWRI, bottke@boulder.swri.edu 
Charles Shearer, Univ. of New Mexico, cshearer@unm.edu

Dimitri A. Papanastassiou, Caltech and JPL, dap@caltech.edu

Carle Pieters, Brown University, carle_pieters@brown.edu

Clive Neal, Univ. of Notre Dame, clive.r.neal.1@nd.edu

Kevin McKeegan, UCLA, mckeegan@epss.ucla.edu

Marc Norman, Australian National University, marc.norman@anu.edu.au

Sarah Valencia, NASA GSFC, sarah.n.valencia@nasa.gov

Kelsey Young, NASA GSFC, kelsey.e.young@nasa.gov

James Tuttle Keane, Caltech/JPL, james.t.keane@jpl.caltech.edu

Heather Meyer, APL, heather.meyer@jhuapl.edu

Maria Banks, GSFC, maria.e.banks@nasa.gov

Mini Wadhwa, ASU,wadhwa@asu.edu

Nicole Schmitz, DLR, nicole.schmitz@dlr.de

Cass Runyon, College of Charleston, runyonc@cofc.edu

Juliane Gross, Rutgers University, jgross@eps.rutgers.edu

Trevor Graff, NASA JSC, trevor.g.graff@nasa.gov

Stephen Elardo, University of Florida, selardo@ufl.edu

Georgiana Kramer, PSI, gkramer@psi.edu

Sara Mazrouei, University of Toronto, sara.mazrouei.seidani@mail.utoronto.ca

Matt Siegler, Planetary Science Institute, msiegler@psi.edu

Kris Zacny, Honeybee Robotics, zacny@honeybeerobotics.com

Michele Faragalli, Mission Control Space Services/Carleton Univ., michele@missioncontrolspaceservices.com

Jessica Flahaut, CNRS/CRPG Nancy, FR flahaut@crpg.cnrs-nancy.fr

Tomas Magna, Czech Geological Survey, Prague, Czech Republic, tomas.magna@geology.cz

Kerri Donaldson Hanna, University of Central Florida, kerri.donaldsonhanna@ucf.edu

R. Aileen Yingst, Planetary Science Institute, yingst@psi.edu

Donald Barker, donald.c.barker@nasa.gov

Csilla Orgel, ESA-ESTEC, csilla.orgel@esa.int

Alan Cassell, NASA Ames Research Center, alan.m.cassell@nasa.gov

Daniel Glavin, NASA GSFC, daniel.p.glavin@nasa.gov

Carolyn Crow, University of Colorado, carolyn.crow@colorado.edu

Paul Hayne, University of Colorado, paul.hayne@lasp.colorado.edu

Leon Alkalai, JPL, leon.alkalai@jpl.nasa.gov

Carolyn van der Bogert, Univ. of Muenster, vanderbogert@uni-muenster.de

Harald Hiesinger, Univ. of Muenster, hhies_01@uni-muenster.de

Gordon “Oz” Osinski, Univ. of Western Ontario, gosinski@uwo.ca

Ralf Jaumann, Freie Universität Berlin, ralf.jaumann@fu-berlin.de 